\title{
Le Calcul des Barrages triangulaires en tenant compte des sous-pressions
}

\author{
Par E. Baticle, Ingnieur en chef des Ponts et Chaussées
}

La question de l'introduction des sous-pressions dans le calcul des barrages a été très discutée. La circulaire du Ministre des Travaux Publics sur les barrages, du 19 octobre 1923, admet qu'on peut négliger cette hypothèse à la condition toutefois que des précautions spéciales soient prises pour empêcher toute infiltration soit dans le corps du barrage, soit à sa base. Mais si on observe quelle minutie exige l'exécution correcte de ces disposilifs spéciaux on peut être conduit à se demander si, tout comple fait, il ne serait pas préférable de calculer le barrage avec l'hypothèse de l'existence des sous-pressions. Le cube de maçonnerie est supérieur, pratiquement, de 15 à $20 \%$, il est vrai ; mais une telle économic doit-elle entrer en ligne de comple quand on songe à l'importance que prennent au point de vue de la sécurité, les efforts d'extension dans un barrage?

Le calcul, d’après la résistance des malériaux, de la stabilité des barrages, avec l'introduction d'une sous-pression s'exerçant suivant un plan horizontal, est classique. Mon intention est de montrer comment on peut faire le calcul par la théorie de l'élasticité, dans le cas d'un barrage triangulaire homogène.

On peut envisager les sous-pressions de deux manières différentes : Ou bien elles s'exercent effectivement dans tout le corps du barrage ; ce serait le cas d'un barrage exécuté avec un matériau poreux, ou bien, le barrage étant lui-mème constitué avec un matériau dépourvu de porosité, bien homogène, la sous-pression s'exerce uniquement sur le sol de fondation. Ce dernier cas sera, évidemment, le plus fréquent. Dans les deux cas, la loi de distribution peut être très variable; j'examinerai l'hypothèse d'une sous-pression égale à la pression hydrostatique (c'est la sous-pression maximum), et celle d'une sous-pression variant suivant une loi linéaire de l'amont à l'aval, égale à la pression hydrostatique à l'amont et nulle à l'aval, comme cela se passerait dans le cas d'un suintement avec une perte de charge par mètre linéaire constante.

$1^{0}$ Sous-pressions dans toute la masse du barrage.- Un élément prismatique infiniment petit de côtés $d x$ et $d y$, est soumis aux forces indiquées sur la figure (1) (l'axe des $y$ est dirigé suivant la verticale descendante).

En écrivant que ces forces sont en équilibre, on a :

$$
\begin{aligned}
& \frac{\partial N_{x}}{\partial x}+\frac{\partial T}{\partial y}=-\frac{\partial p}{\partial x} \\
& \frac{\partial \mathrm{T}}{\partial x}+\frac{\partial N_{\mathrm{y}}}{\partial y}=\pi-\frac{\partial p}{\partial y}
\end{aligned}
$$

On a une intégrale dépendant de quatre constantes arbitraires en prenant :

$$
\begin{aligned}
\mathrm{N}_{\mathrm{x}} & =\alpha x+\beta y-p \\
\mathrm{~N}_{\mathrm{y}} & =x^{\prime} x+\beta^{\prime} y-p \\
\mathrm{~T} & =\left(\pi-\beta^{\prime}\right) x-\alpha y
\end{aligned}
$$

On détermine les 4 constanles en écrivant que sur les parements, les composantes de la force moléculaire sont égales aux pressions supportées par les dits parements.
On sait que sur une droite inclinée de $\theta$ l'effort moléculaire a pour composantes :

$$
\begin{aligned}
& X=N_{x} \sin \theta-T \cos \theta \\
& Y=T \sin \theta-N_{y} \cos \theta
\end{aligned}
$$

On a ainsi, pour chaque parement, 2 équations. C'est la méthode classique : elle s'applique dans le cas présent si $p$ est de la forme $: p=a x+b y$.

Je vais considérer le cas simple d'un barrage à parement amont vertical, $i$ étant l'inclinaison du parement aval. J'appelle $k$ le poids du mètre cube d'eau. On a pour : $x=0$ :

D’où :

$$
\mathrm{N}_{\mathrm{x}}=\mathrm{K} y \text { et } \mathrm{T}=0 \text {. }
$$

et pour : $y=x t g i$ :

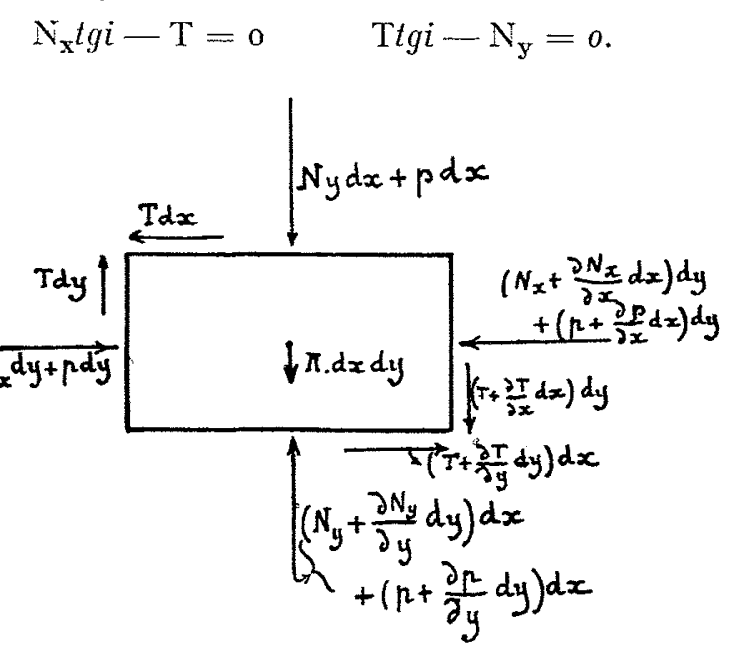

Fig. 1

On lire facilement de ces équations :

$$
\begin{aligned}
& x^{\prime}=-\pi t g i+2 K t g^{3} i-2 a t g^{2} i+b t g i \\
& \beta^{\prime}=\pi-k t g^{2} i+a t g i .
\end{aligned}
$$

Supposons la sous-pression uniforme et égale à la pression hydrostatique. On aura : $\quad a=o \quad b=\mathrm{K}$.

$$
\text { D'où : }\left\{\begin{array}{l}
\mathrm{N}_{\mathrm{x}}=\mathrm{K} y \\
\mathrm{~N}_{\mathrm{y}}=\left[2 \mathrm{~K} t g^{3} i-(\pi-\mathrm{K}) \operatorname{tg} i\right] . x+\left(\pi-k-k \operatorname{tg}^{2} i\right) y \\
\mathrm{~T}=\mathrm{K} x t g^{2} i .
\end{array}\right.
$$

La condition pour qu'il n'y ait pas d'effort d'extension sur le parement amont est :

$$
\begin{gathered}
\pi-k-k \operatorname{tg}^{2} i \bar{y} 0 \\
\text { ou } \operatorname{tg} i=\sqrt{\frac{\pi-\mathrm{K}}{\mathrm{K}}} .
\end{gathered}
$$

Tout se passe d'ailleurs dans ce cas comme si la densité du barrage était uniformément diminuée de $K$.

Supposons maintenant que la sous-pression, égale à la pression hydrostatique sur le parement amont, soit nulle sur le parement aval; on a alors : $b=\mathrm{K}$ et $a+b t g i=o$ d'où $: a=-\mathrm{K}$ tgi. Les expressions des efforts moléculaires deviennent:

$$
\begin{aligned}
\mathrm{N}_{\mathrm{x}} & =\mathrm{K} y \\
\mathrm{~N}_{\mathrm{y}} & =\left[4 \mathrm{~K} t g^{3} i-(\pi-\mathrm{K}) \lg i\right] x+\left(\pi-\mathrm{K}-2 \mathrm{~K} t g^{2} i\right) y \\
\mathrm{~T} & =\mathrm{K} x g^{2} i .
\end{aligned}
$$


Pour qu'il n'y ait pas d'effort d'extension sur le parement amont il faut et il suffit que l'on ail :

$$
\begin{aligned}
& \text { - - K }-2 \mathrm{~K}^{2} g^{2} i \bar{\gamma} \\
& \text { ou : } \lg i=\sqrt{\frac{\pi-\mathrm{K}}{2 \mathrm{~K}}}
\end{aligned}
$$

On voit que cette hypothèse est beancoup plus défavorable que l'hypothèse de la sous-pression égale en tous points à la pression hydrostatique. Ce calcul montre le très grand intérèt que présente, au point de vue de la sécurité, la compacité du matériau, d'où dépend l'imperméabilité du barrage.

$2^{\circ}$ Sous-pression ne s'exercant que dans un plan horizontal. C'est le cas, ai-je dit plus haut, d'un barrage exécuté avec un matériau rigoureusement imperméable, mais où néanmoins des injiltrations restent possibles, entre la fondation et le lerrain d'assise.

Je supposerai tonjours que la sous-pression est linéaire et de la forme :

$$
p=a x+b y_{0} .
$$

$y_{\mathrm{n}}$ étant la hauteur du barrage (fig. 3).

Tout se passe comme si chaque branche $\mathrm{A} a$, d'épaisseur $d x$ avait sa densité diminuée de $\frac{\mathrm{P}}{y_{\mathrm{o}}-x t g i_{\mathrm{o}}}$ dans la partieà l'aval de oy, et de $\frac{\mathrm{P}}{y_{\mathrm{o}}+x t g \dot{i}_{1}}$ dans la partie à l'amont de $o y$.

Dans ce cas, les équations indéfinies d'équilibre sont :

$$
\begin{gathered}
\frac{\partial \mathrm{N}_{\mathrm{x}}}{\partial x}+\frac{\partial \mathrm{T}}{\partial y}=0 \\
\frac{\partial \mathrm{T}}{\partial x}+\frac{\partial \mathrm{N}_{\mathrm{y}}}{\partial y}=\pi-\frac{a x+b y_{\mathrm{o}}}{y_{\mathrm{o}}-x \operatorname{tg} i_{\mathrm{o}}}
\end{gathered}
$$

pour la partie à l'aval de $\mathrm{O} y$ et un système analogue ou $i_{\mathrm{o}}$ est remplacée par $-i_{1}$ pour la partie à l'amont de oy.

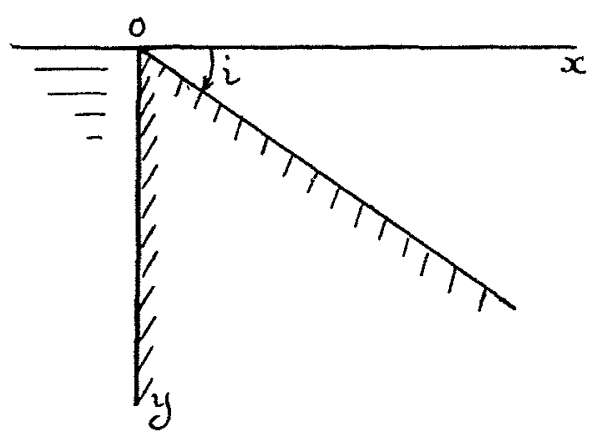

Fig. 2

On aura la solution de ces systèmes dépandant de 4 constantes et d'une fonction arbitraires en prenant :

$$
\begin{aligned}
& \mathrm{N}_{\mathrm{x}}=\alpha x+\beta y \\
& \mathrm{~N}_{\mathrm{y}}^{\mathrm{l}}=\alpha^{\prime} x+\beta^{\prime} y-y \frac{b y_{\mathrm{o}}+a x}{y_{\mathrm{o}}-x \operatorname{lo} i_{\mathrm{o}}}+f_{\mathrm{o}}(x) \\
& \mathrm{T}=\left(\pi-\beta^{\prime}\right) x-\alpha y \\
& \text { et }\left\{\begin{array}{l}
\mathrm{N}_{\mathrm{x}}=\alpha x+\beta y \\
\mathrm{~N}_{\mathrm{y}}=\alpha^{\prime} x+\xi^{\prime} y-y \frac{a x+b y y_{\mathrm{o}}}{y_{\mathrm{o}}+x \lg i_{1}}+f_{1}(x) \\
\mathrm{T}=\left(\pi-\beta^{\prime}\right) x-\alpha y
\end{array}\right.
\end{aligned}
$$

On pourra déterminer $f_{\mathbf{0}}(x)$ et $f_{1}(x)$ de façon que les termes non linéaires s'annulent aux frontières, c'est-à-dire, respectivement, pour $y=x l g i_{\mathrm{o}}$ et $y=-x l g i_{1}$. D'où :

$$
\begin{aligned}
& f_{\mathrm{o}}(x)=x \operatorname{tg} i_{\mathrm{o}} \frac{a x+b y_{\mathrm{o}}}{y_{\mathrm{o}}-x \lg i_{\mathrm{o}}} \\
& f_{1}(x)=-x t g i_{1} \frac{a x+b y_{\mathrm{o}}}{y o_{\mathrm{o}}+x t g i_{1}}
\end{aligned}
$$

Les termes non linéaires auront ainsi la forme suivante :

$$
\frac{y-x t g i_{0}}{y_{0}-x t g i_{1}}\left(a x+b y_{0}\right) \text { et } \frac{y+x t g i_{1}}{y_{0}+x t g i_{1}}\left(a x+b y_{0}\right)
$$

Si on remarque que leurs valeurs sont égales pour $x=0$; ce qui fait que, quels que soient les $(\alpha, \beta)$ les valeurs des $(\mathrm{N}, \mathrm{T})$ sont égales sur oy, il suffira, pour achever de déterminer les efforts intérieurs, d'écrire les 4 équations exprimant les conditions surr les parements :

$$
\begin{aligned}
& \mathrm{N}_{\mathbf{x}} t g i_{\mathrm{o}}-\mathrm{T}=0 \\
& \mathrm{~T} t g i_{\mathbf{o}}-\mathrm{N}_{\mathrm{y}}=0 \quad \text { pour } \quad y=x t g i_{\mathbf{o}} \\
& \mathrm{N}_{\mathrm{x}} t g i_{1}+\mathrm{T}=\mathrm{K} y t g i_{1} \\
& \mathrm{~T} t g i_{1}+\mathrm{N}_{\mathrm{y}}=\mathrm{K} y \quad \text { pour } \quad y=t g i_{\mathbf{1}} .
\end{aligned}
$$

On retrouve, en définitive, les mêmes $(\alpha, \beta)$ que s'il n'y avait pas de sous-pression. Seule $\mathrm{N}_{\mathbf{y}}$ est diminuée du terme complé-

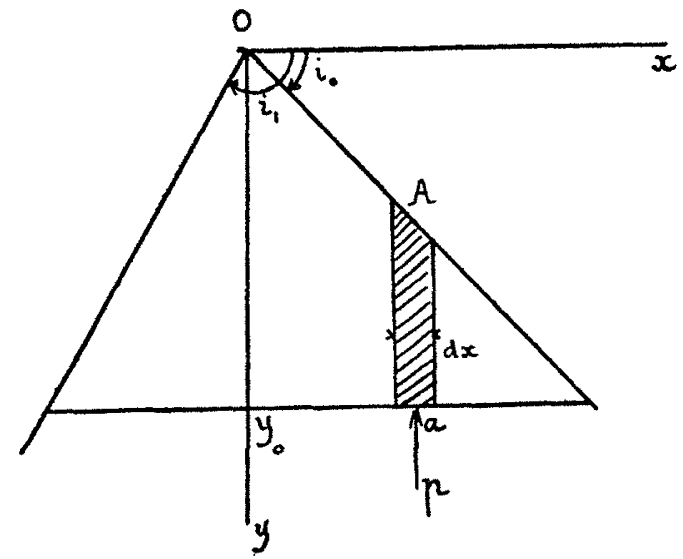

lig. :3

mentaire, qui varie linéairement sur une verticale, à partir du parement, où il est nul, jusqu'à la base, où il est égal à la souspression.

Comme précédemment, je vais expliciter les formules don nant les $N$ et $T$ pour le cas simple d'un barrage à parement amont vertical.

On a :

$$
\begin{aligned}
\mathrm{N}_{\mathrm{x}} & =x x+\beta y \\
\mathrm{~N}_{\mathrm{y}} & =\alpha^{\prime} x+\xi^{\prime} y-\frac{y-x t g i}{y_{\mathrm{o}}-x t g i}\left(a x+b y_{\mathrm{o}}\right) \\
\mathrm{T} & =\left(\pi-\beta^{\prime}\right) x-\alpha l
\end{aligned}
$$

pour $x=0 \quad \mathrm{~N}_{\mathbf{x}}=\mathrm{K} y \quad$ el $\quad \mathrm{T}=0 . \quad$ D'où $: \beta=\mathrm{K} \quad \alpha=0$ pour : $y=x l g i \quad \mathrm{~N}_{\mathbf{x}}^{1} t g i-\mathrm{T}=0$

et

$$
\mathrm{T} t g i-\mathrm{N}_{\mathrm{y}}=0
$$

c'est-à-dire:

$$
\mathrm{K} t g^{2} i-\left(\pi-\beta^{\prime}\right)=0
$$

D'où :

$$
\left(\pi-\beta^{\prime}\right) \lg i-\alpha^{\prime}-\xi^{\prime} \operatorname{tg} i=0 .
$$

$$
\beta^{\prime}=\pi-\mathrm{K} t g^{2} i
$$$$
\alpha^{\prime}=\left(\pi-2 \xi^{\prime}\right) t g i=\left(2 \mathrm{~K} l q^{2} i-\pi\right) t g i .
$$

On a par conséquent :

$\mathrm{N}_{\mathbf{x}}=\mathrm{K} y$

$\mathrm{N}_{\mathrm{y}}=\left(2 \mathrm{~K} l g^{2} i-\pi\right) \lg i x+\left(\pi-\mathrm{K} l g^{2} i\right) y-\frac{y-x l g i}{y_{\mathrm{o}}-x \lg i}\left(a x+b y y_{0}\right)$ $\mathrm{T}=\mathrm{K} \ell^{2} i . x$

La condition de non-extension sur le parement amont $(x=0)$ est :

$$
\pi-\mathrm{K} l g^{2} i-b>0
$$

Si $b=\mathrm{K}$, on relrouve, quel que soil $a$, la condition de Maurice Lévy :

$$
\lg i=\sqrt{\frac{\pi-\mathrm{K}}{\mathrm{K}}}
$$

